\title{
Self-hardening of Nanocrystalline Ti-B-N Thin Films
}

\author{
J. G. Wen, ${ }^{*}$ P. H. Mayrhofer, ${ }^{* *}$ C. Mitterer, ${ }^{* *}$ J. E. Greene, ${ }^{*}$ and I. Petrov* \\ *Frederick Seitz Materials Research Laboratory, University of Illinois, Urbana, IL 61801 \\ **Department of Physical Metallurgy, University of Leoben, A-8700 Leoben, Austria
}

Nanocrystalline hard films have attracted increasing interest for wear-resistant applications. We study the ternary system Ti- B- N which offers advantages, due to the large number of equilibrium and metastable phases as a model system for investigating nanostructural effects on film hardness. Using magnetron sputtering we have grown nanocrystalline films, with an overall composition of $\mathrm{TiB}_{0.80} \mathrm{~N}_{0.83}$ which in the equilibrium phase diagram corresponds to mole fractions of $51.5 \% \mathrm{TiN}$, $37.0 \% \mathrm{TiB}_{2}$, and $11.5 \% \mathrm{BN}$. The as-deposited layers have a high nanoindentation hardness $H$ of 37 $\mathrm{GPa}$. However, as opposed to binary carbides and nitrides for which $H$ typically decreases at elevated temperatures $T_{a}$ due to a reduction in defect density, the hardness of our films actually increases to $43 \mathrm{GPa}$ at $T_{a}=800{ }^{\circ} \mathrm{C}$. Thus, the high and superhigh hardness of these layers are not due to intrinsic growth stresses but due to nanostructure evolution which we study using a combination of $\mathrm{x}$-ray diffraction, electron-probe microanalysis (EPMA), and cross-sectional transmission electron microscopy (XTEM).

Bright-field, Z-contrast (Fig. 1), high-resolution XTEM (Fig. 2a) and SAED (Figure 3a) show that films grown at $300{ }^{\circ} \mathrm{C}$ consist of a high volume fraction, $\sim 50 \%$, of a fully percolated disordered $\mathrm{BN}$ rich phase encapsulating randomly-oriented 2- $3 \mathrm{~nm}$ wide TiN and TiB2 grains. During vacuum annealing at $T_{a}=800{ }^{\circ} \mathrm{C}$ the $\mathrm{B} / \mathrm{Ti}$ composition ratio and the average grain size $d$ remain constant at 0.80 and $2-3 \mathrm{~nm}$, respectively, while a structural rearrangement of the disordered phase results in the formation of compact interface boundaries which lead, in turn, to the observed hardness increase. EPMA results show that the N/Ti ratio in the films remains constant at $\sim 0.83$ up to the highest annealing temperatures, $1100{ }^{\circ} \mathrm{C}$, used in these experiments.

At $900{ }^{\circ} \mathrm{C}$ the average grain size increased slightly to $\sim 4 \mathrm{~nm}$ (Fig. 2c). The crystals are larger than $10 \mathrm{~nm}$ after annealing at $T_{a}=1100^{\circ} \mathrm{C}$ (Fig. 2d). The SAED investigations of the films after different annealing treatments (Fig. 3b-3d) show increasingly sharper diffraction rings in agreement with the grain growth observed by HRXTEM. However, there is no evidence in either XRD or SAED results for the presence of crystalline BN. Dynamical DSC measurements of Ti-B-N films, removed form their substrates, coupled with EPMS data, show loss of B, by the formation of volatile B-oxides with residual oxygen. This is consistent with the thickness change after annealing as shown in Fig. 4a. Annealing at $T_{a}=900{ }^{\circ} \mathrm{C}$ results in B loss by evaporation from the disordered phase, and hence the

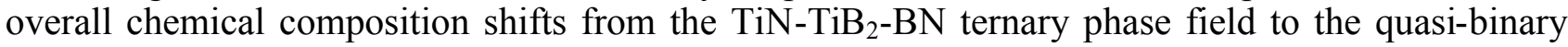
$\mathrm{TiN}-\mathrm{TiB}_{2}$ tie line, and an increase in crystallite size. The hardness decreases at higher annealing temperatures as the probability for dislocation formation increases with larger grain size. However, boundary layer compaction and grain growth result in the elastic modulus E continuing to increase with $T_{a}$ since the disordered regions have lower elastic constants than crystallites.

The overall results provide insight toward developing "design rules" for a new class of nano-scale based coatings in which the hardness and the elastic modulus actually increase with temperature.

We acknowledge the use of the Center for Microanalysis of Materials, University of Illinois. J.G.W, I.P., and J.E.G. are supported by U.S. Department of Energy, under Award No. DEFG0291ER45439. The Erwin Schrödinger Program (project J2469- N02) of the Austrian Science Fund (FWF) is acknowledged by P.H.M for financial support. 


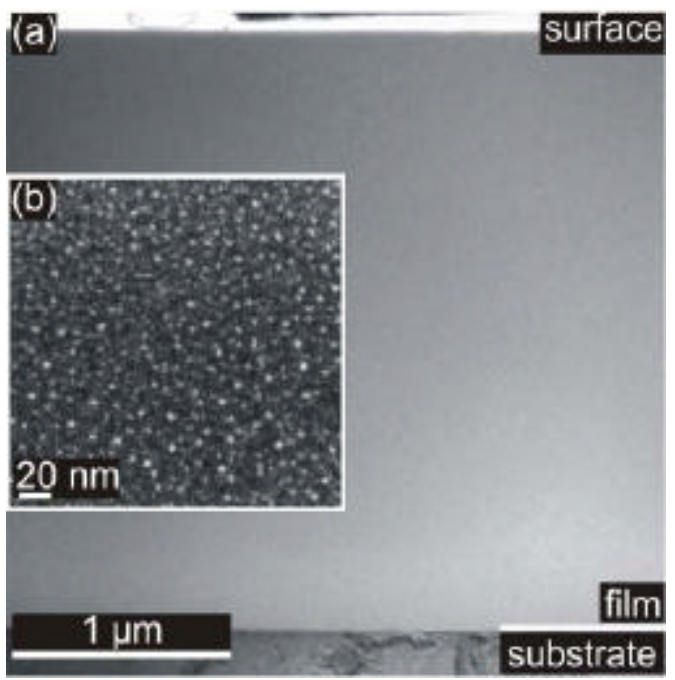

Fig. 1 (a) Cross-sectional bright field TEM image from an as-deposited nanocrystalline Ti- B- N film, (b) Zcontrast image indicating at least two different phases. Brighter regions correspond to a phase composed of higher $\mathrm{Z}$ elements than the darker regions.
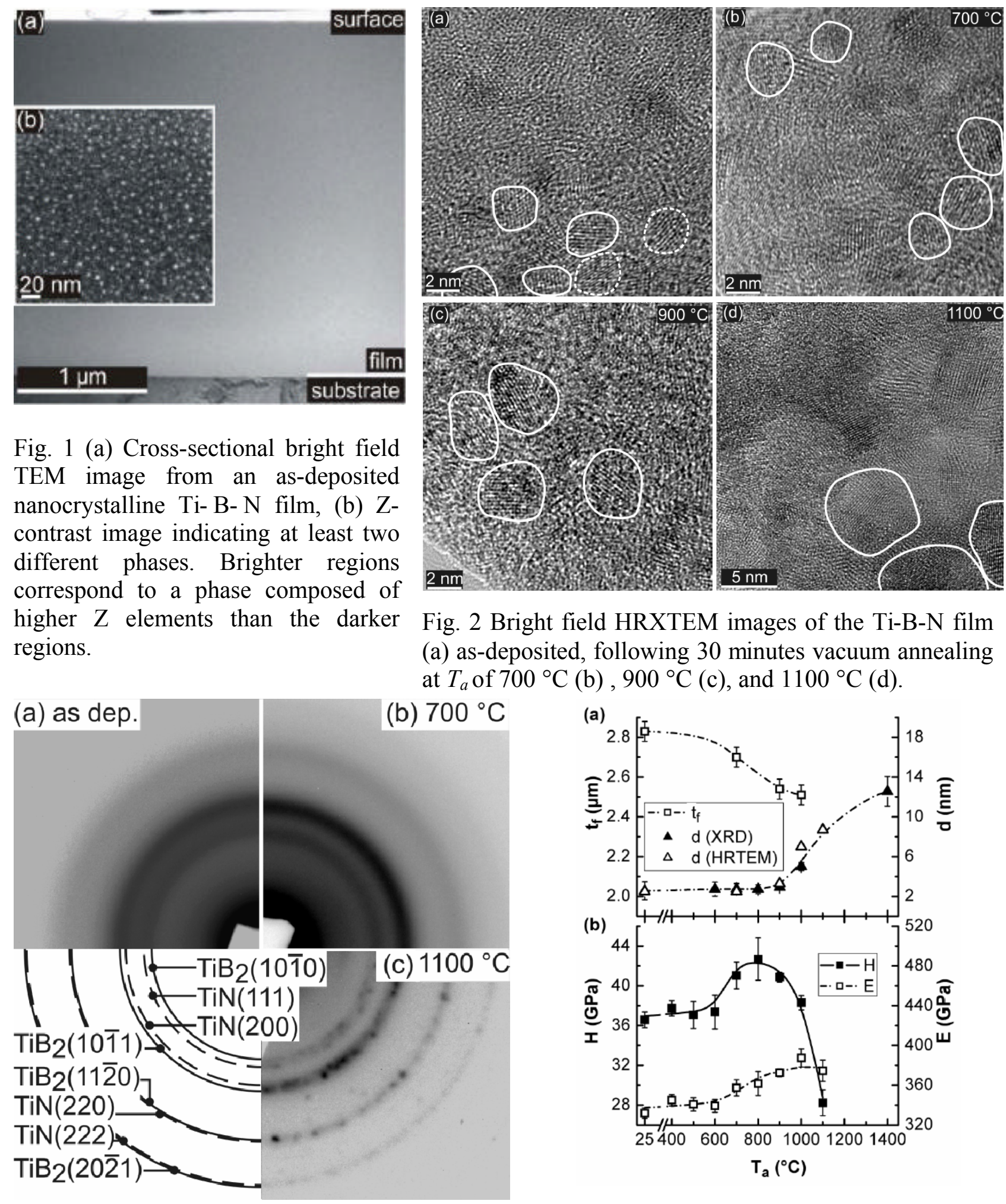

Fig. 2 Bright field HRXTEM images of the Ti-B-N film (a) as-deposited, following 30 minutes vacuum annealing at $T_{a}$ of $700{ }^{\circ} \mathrm{C}(\mathrm{b}), 900{ }^{\circ} \mathrm{C}(\mathrm{c})$, and $1100{ }^{\circ} \mathrm{C}$ (d).

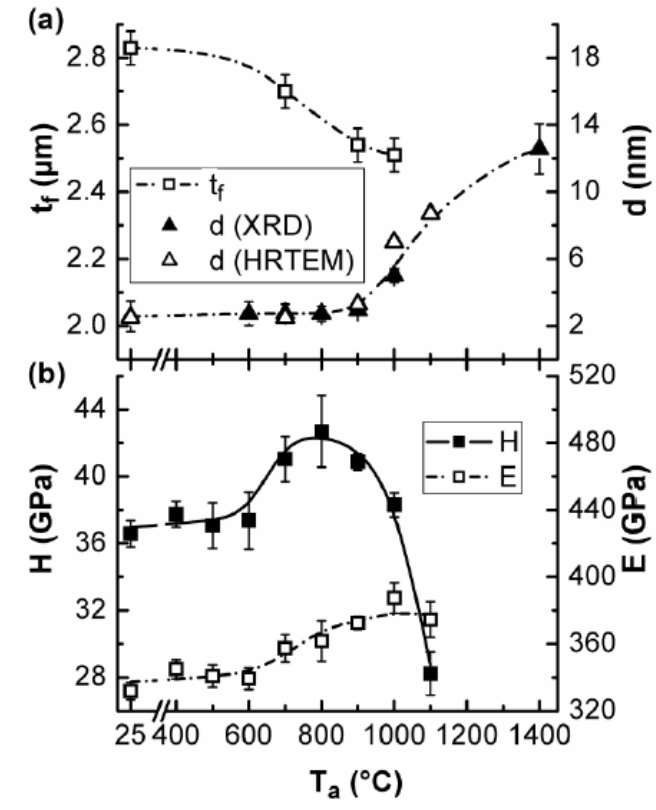

Fig. 3 Development of the SAED diffraction rings of Ti-B-N with $T_{a}$, the standard diffraction rings for fcc-TiN and hcp- $\mathrm{TiB}_{2}$ are added.

Fig. 4 (a) Film thickness $t_{f}$ and average grain sizes $d$. (b) Hardness $H$ and nanoindentation moduli $E$ of Ti-B-N layers as a function of $T_{a}$. 